How not to smoke like a chimney

\section{OP0217-HPR EVALUATION OF PATIENTS' EXPERIENCES OF AN INTENSIVE SMOKING CESSATION INTERVENTION FOR PEOPLE WITH RHEUMATOID ARTHRITIS}

Ida Kristiane Roelsgaard ${ }^{1}$, Thordis Thomsen ${ }^{2,3}$, Mikkel Østergaard ${ }^{3,4}$, Anne Grete Semb ${ }^{5}$, Lena Andersen ${ }^{6}$, Bente Appel Esbensen ${ }^{3,4} .{ }^{1}$ Rigshospitalet, Center for Rheumatology and Spine Diseases, Copenhagen Center for Arthritis Research (COPECARE), Glostrup, Denmark; ${ }^{2}$ Copenhagen University Hospital HerlevGentofte, Herlev Anaesthesia Critical and Emergency Care Science Unit, ACES, Department of Anesthesiology, Herlev, Denmark; ${ }^{3}$ University of Copenhagen, Department of Clinical Medicine, Faculty of Health and Medical Sciences, Copenhagen, Denmark; ${ }^{1}$ Rigshospitalet, Center for Rheumatology and Spine Diseases, Copenhagen Center for Arthritis Research (COPECARE), Glostrup, Denmark; ${ }^{5}$ Diakonhjemmet Hospital, Preventive Cardio-Rheuma Clinic, Department of Rheumatology, Oslo, Norway; ${ }^{6}$ The Danish Rheumatism Association, Gentofte, Denmark

Background: Smoking is considered one of the most important modifiable environmental risk factors to the pathogenesis of rheumatoid arthritis (RA). Smoking may also exacerbate the symptoms of RA. A randomized controlled trial (RCT) testing the effect of an intensive smoking cessation intervention on smoking cessation and disease activity in people with RA is currently being carried out (1).

Objectives: To evaluate how people with RA experienced an intensive smoking cessation intervention.

Methods: We conducted a qualitative study with individual interviews. Participants were recruited consecutively from the intervention group in a smoking cessation RCT after having completed both the smoking cessation intervention and the three-month follow-up visit. The analysis was based on thematic analysis (2). One patient research partner was involved in all phases of the study.

Results: In total 12 patients with RA were included ( 6 female, mean age 58 years (range 33-71)). We identified seven themes illustrating participants' experiences of the smoking cessation intervention. The themes were; Instilling hope for smoking cessation referring to the initial invitation to participate in the RCT and the allocation to the intervention group, which participants seized as a chance to stop smoking, Having a fellow traveler on the road to smoking cessation referring to their cooperation with the smoking cessation counselor which was important for maintaining motivation for smoking cessation, Tangible evidence that smoking cessation makes a difference referring to improved carbon monoxide levels which motivated endeavors to quit smoking, Apprehension - fear of a new dependence referring to participants' fear of becoming addicted to nicotine replacement therapy instead of smoking, Breaking habits referring to ongoing reflection on and efforts to quit smoking using the tools and knowledge obtained from the smoking cessation intervention, Why wasn't I told? Referring to the lacking provision of information on smoking and RA from health professionals, and Denial referring to neglect of the detrimental impact of smoking on RA symptoms and overall health.

Conclusion: The participants were grateful for the offer to participate in a RCT and a smoking cessation intervention because it created an opportunity for them to stop smoking. They valued getting concrete information about detrimental effects of smoking on RA and RA treatment. Many requested that health professionals focus more proactively on smoking and smoking cessation.

\section{REFERENCES:}

[1] Roelsgaard et al. The effect of an intensive smoking cessation intervention on disease activity in patients with rheumatoid arthritis: study protocol for a randomised controlled trial. Trials. 2017;18(1):570.

[2] Braun V et al. Using thematic analysis in psychology. Qualitative Research in Psychology. 2006;3(2):77-101.

Disclosure of Interests: Ida Kristiane Roelsgaard: None declared, Thordis Thomsen: None declared, Mikkel Østergaard Grant/research support from: Abbvie, Celgene, Centocor, Merck, Novartis, Consultant for: Abbvie, BMS, Boehringer-Ingelheim, Celgene, Eli Lilly, Hospira, Janssen, Merck, Novartis, Novo, Orion, Pfizer, Regeneron, Roche, and UCB, Speakers bureau: Abbvie, BMS, Boehringer-Ingelheim, Celgene, Eli Lilly, Hospira, Janssen, Merck, Novartis, Novo, Orion, Pfizer, Regeneron, Roche, and UCB, Anne Grete Semb: None declared, Lena Andersen: None declared, Bente Appel Esbensen Speakers bureau: For Pfizer

DOI: 10.1136/annrheumdis-2019-eular.4362
THURSDAY, 13 JUNE 2019

\section{Should we worry about anything else - also young people have multi-morbidities}

\section{OP0218-PARE PREVENTATIVE MEDICINE THROUGH IDENTIFYING AND ADDRESSING COMORBIDITIES OF SPONDYLOARTHRITIS (SPA*) TO IMPROVE PATIENT OUTCOMES THROUGH PATIENT ORGANIZATION PARTNERSHIPS AND ALLIED HEALTHCARE OUTREACH}

Wendy Gerhart ${ }^{1}$, Gerald Major ${ }^{2}$, Austin Gerhart ${ }^{2}$, Marilyn Walsh ${ }^{2} .{ }^{1}$ Canadian Spondylitis Association, Phelpston, Canada; ${ }^{1}$ Canadian Spondylitis Association, Phelpston, Canada

Background: Many patients are not aware of the common comorbidities they may experience. We need to understand if by educating patients and managing or preventing comorbidities, will patient outcomes be improved and the impact on healthcare system.

Objectives: To determine what type and number of comorbidities patients commonly experience in addition to their diagnosed SpA condition. This will help identify how collaboration with other patient organizations and allied healthcare professionals can better raise awareness, educate and support the community. It will convey the conditions, symptoms and importance of early diagnosis and treatment. This will reduce the impact on the Canadian healthcare system and improve patient outcomes

Methods: Canadian patients were surveyed from February through June 2018. Respondents were asked a variety of questions to gain insights on the comorbidities and how many they experience and live with in addition to their SpA diagnosis.

Results: The most common comorbidities respondents reported included: chronic pain (55\%), mental health (depressions \& anxiety) (54\%), gut (Crohn's and ulcerative colitis)(35\%), eye inflammation (uveitis/iritis) (33\%), sleep issues $(30 \%)$ and chronic fatigue $(26 \%)$. Interestingly, these results are different from other studies where osteoporosis and cardiovascular disease were most commonly experienced. In addition to the more prevalent comorbidities, a significant number of people experienced skin (psoriasis), fibromyalgia, hypertension and lung disease.

There were 406 respondents who completed the survey. Only 3.7\% reported to not have any comorbidities.

$42.6 \%$ of patients were diagnosed with another condition in advance of being diagnosed with a SpA disease. There were $44.6 \%$ of respondents who had a healthcare professional other than their GP or Rheumatologist suggest they may have a SpA condition. Recommendation for further assessment was initiated by a physiotherapist $(12 \%)$, a chiropractor $11 \%)$, ophthalmologist $(7 \%)$ or massager therapist $(5 \%)$.

Conclusion: A significant number of patients diagnosed with a SpA condition experience comorbidities and often have more than one condition. On average people live with 4 different comorbidities. The survey results have confirmed the significance depression and anxiety impacts patients with SpA.

Through partnerships with trusted patient organizations, we can provide resources to educate and support our SpA community on the comorbidities commonly experienced. This will empower patients to manage symptoms sooner, thus contributing to a quicker recovery, reduced damage or prevent the complication from occurring all together.

Expanding outreach and collaborating with allied healthcare professionals through Canadian professional associations including family physicians, physiotherapists, chiropractors, ophthalmologists and massage therapists can further educate practicing healthcare professionals on SpA conditions and symptoms to recognize with patients contributing to prevention or earlier diagnosis and treatment intervention

The CSA will form collaborative agreements by leveraging existing partnerships and creating new partnerships with Canadian organizations and professional associations to support and educate people with credible and valuable resources on the comorbidities identified through this survey with the intended purpose to treat earlier or prevent complications that adds unnecessary financial strain on the Canadian healthcare system.

Together we are stronger.

REFERENCES:

[1] Molto, A., Nikiphorou, E. Comorbidities in Spondyloarthritis. Frontiers in Medicine. March 2018. Volume 5. Article 62

[2] Danve, A., Raychaudhuri, S. Comorbidities in Spondyloarthritis. Curr Treat Options in Rheum (2017) 3:63-74 